\title{
An ATP-binding Cassette Multidrug-Resistance Transporter Is Necessary for Tolerance of Gibberella pulicaris to Phytoalexins and Virulence on Potato Tubers
}

\author{
André Fleißner, Claudia Sopalla, and Klaus-Michael Weltring \\ Institut für Botanik, Westfälische Wilhelms-Universität, Schlossgarten 3, 48149 Muenster, Germany \\ Submitted 13 July 2001. Accepted 23 October 2001.
}

\begin{abstract}
The necrotrophic pathogen Gibberella pulicaris infects potato tubers through wounds that contain fungitoxic secondary metabolites such as the phytoalexins rishitin and lubimin. In order to colonize tuber tissue, the fungus must possess a mechanism to tolerate potato defense compounds. In this paper, we show that a gene, Gpabc1, that codes an ATP-binding cassette $(\mathrm{ABC})$ transporter is required for tolerance to these phytoalexins and for virulence on potato. The Gpabc1 gene, isolated in the course of a differential cDNA screen, shares high sequence homology with the ABC1 gene of Magnaporthe grisea. G. pulicaris mutants deficient in Gpabc1 were still able to metabolize rishitin but lost their tolerance to this phytoalexin as well as their virulence on potato. These results strongly suggest that the Gpabc1-encoded ABC transporter is necessary for tolerance of $G$. pulicaris to rishitin and that this tolerance is required for virulence on potato.
\end{abstract}

Since the term "phytoalexin" was introduced by Müller and Börger about 60 years ago (Müller and Börger 1940), the production of antimicrobial secondary metabolites by plants in response to wounding and microbial infection has been considered a major defense mechanism of plants. Phytoalexins constitute a structurally diverse group of chemicals, including phenylpropanoids, terpenoid, and fatty acid derivatives, and they have been found to accumulate to high levels in damaged tissue of many plants (Ebel 1986). In the 1960s, work by Cruickshank (1962; Cruickshank and Perrin 1965) indicated that tolerance to phytoalexins might be an important requirement for phytopathogenic fungi. Since then, much effort has been invested in research involving the detoxification of phytoalexins by degradation (Morrissey and Osbourn 1999; VanEtten et al. 1995). Genetic studies with Nectria haematococca (Miao and VanEtten 1992; VanEtten et al. 1989) and Gibberella pulicaris (Desjardins and Gardner 1989, 1991; Desjardins et al. 1992) supported the correlation between the ability of these fungi to metabolize their host phytoalexin and virulence on their host plants.

The application of molecular genetic techniques to phytopathogenic fungi led to the isolation of two genes from $N$. haematococca that code for phytoalexin-detoxifying enzymes. The $P D A 1$ gene coding for pisatin demethylase and the $M A K 1$ gene coding for maackiain 1a-hydroxylase are involved in detoxifi-

Corresponding author: K.-M. Weltring; Telephone: 0049-251-8324976; Fax: 0049-251-8321601; E-mail: weltrin@uni-muenster.de cation of the phytoalexins pisatin from pea and maackiain from chickpea, respectively (Covert et al. 1996; Weltring et al. 1988). Disruption mutants of both genes showed reduced virulence on pea and chickpea, indicating that metabolism of phytoalexins to less toxic products contributes to, but is not essential for, virulence of $N$. haematococca on these host plants (Enkerli et al. 1998, Wasmann and VanEtten 1996). These data raised the possibility that other mechanisms of phytoalexin tolerance might be important for virulence of phytopathogenic fungi.

The necrotrophic fungus G. pulicaris (anamorph Fusarium sambucinum) causes potato dry rot disease by infecting tubers through wounds. Since wounding of potato tubers has been shown to induce production of phytoalexins, especially rishitin and lubimin, the fungus is likely to be exposed to high levels of these phytoalexins during infection (Kuc 1982). Genetic studies indicated a correlation between tolerance to rishitin and virulence of the fungus (Desjardins and Gardner 1989, 1991; Desjardins et al. 1992). This correlation made G. pulicaris an ideal candidate to identify rishitin-induced genes involved in phytoalexin tolerance via a differential cDNA screen.

ATP-binding cassette (ABC) multidrug-resistance transporters, which have been found in all prokaryotic and eukaryotic organisms examined so far, are a likely candidate for an alternative tolerance mechanism. Most ABC transporters in higher eukaryotes consist of two transmembrane domains (TMD), each containing six membrane-spanning regions and two nucleotide binding folds (NBF) in a two times two domain configuration. The transporter proteins can have either a $[\mathrm{TMD}-\mathrm{NBF}]_{2}$ or an $[\mathrm{NBF}-\mathrm{TMD}]_{2}$ configuration, depending on the location of the NBF. In addition, $\mathrm{ABC}$ transporters are characterized by the presence of a Walker A and Walker B motif separated by 120 to 170 amino acids, including an ABC signature (Walker et al. 1982; Hyde et al. 1990).

By definition, multidrug-resistance $\mathrm{ABC}$ transporters have a broad substrate range. The best characterized examples are the yeast Pdr5p and the human MDR1 transporters (Balzi et al. 1994; Endicott and Ling 1989). MDR1 has 93 known substrates of various chemical classes (Gottesman and Pastan 1993). The physiological role of these transporters is the active secretion of toxic substances in order to protect the cell from damage by these compounds. This function may be especially important for plant pathogenic fungi, as proposed by De Waard (1997) and Del Sorbo and associates (2000), because they may provide protection against fungitoxic defense compounds produced by plants in response to infection. This hypothesis is supported by the observation that a wide variety of natural compounds can act as substrates or inducers of $\mathrm{ABC}$ transport- 
ers (del Sorbo et al. 1997; Gottesman and Pastan 1993; Seelig 1998) and by the reduced virulence on grapevine and the increase of sensitivity to the grapevine phytoalexin resveratrol of mutants of Botrytis cinerea in which the ABC transporter BcatrB had been disrupted (Schoonbeek et al. 2001). The latter result in particular suggests that $\mathrm{ABC}$ transporters can contribute to virulence of phytopathogenic fungi by reducing the toxic effect of phytoalexins.

Additional indirect evidence for the role of membrane transporters in phytoalexin tolerance was provided by the presence of an energy-dependent, nondegradative tolerance mechanism against the pea phytoalexin pisatin in N. haematococca involving reduced retention of pisatin in the mycelium (Denny and VanEtten 1983a, 1983b; Denny et al. 1987).

In this paper, we describe the isolation of a gene encoding a multidrug-resistance $\mathrm{ABC}$ transporter by differential cDNA screening. Replacement of the gene in G. pulicaris dramatically reduced tolerance to potato phytoalexins as well as virulence on potato tubers. These data demonstrate that multidrugresistance transporters are necessary for both tolerance to phytoalexins and pathogenicity of $G$. pulicaris on potato tubers.

\section{RESULTS}

\section{Isolation and characterization of the Gpabc1 gene.}

A cDNA library in $\lambda$-ZAP prepared from RNA isolated from mycelium exposed to rishitin on agar plates for 2 to $4 \mathrm{~h}$ was screened with radiolabeled cDNA from rishitin-induced $(2 \mathrm{~h})$ and noninduced mycelia. Two partial cDNA clones with homology to multidrug-resistance $\mathrm{ABC}$ transporters of other fungi were obtained and used as probes to isolate full-length cDNAs and genomic sequences from a cosmid library. Sequencing of these DNA fragments and comparison of the genomic sequence with that of the cDNAs confirmed that both isolated partial cDNA clones belonged to the same 4,576-bp open reading

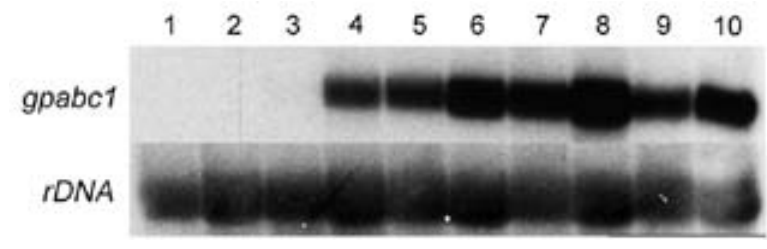

Fig. 1. Time course of the induction of Gpabcl. The expression of Gpabcl after adding rishitin $(150 \mu \mathrm{g} / \mathrm{ml})$ to liquid cultures was tested by northern analysis using total RNA. Lane 2, 0 min; lane 3, 5 min; lane 4, $10 \mathrm{~min}$; lane 5, $15 \mathrm{~min}$; lane 6, $20 \mathrm{~min}$; lane 7, $30 \mathrm{~min}$; lane 8, $40 \mathrm{~min}$; lane 9, $50 \mathrm{~min}$; and lane 10, $60 \mathrm{~min}$ after adding rishitin. Lane 1, untreated control, $30 \mathrm{~min}$ incubation. The equal loading of each lane with $15 \mu \mathrm{g}$ of RNA per lane was verified by hybridization with a part of the rDNA cluster of Gibberella pulicaris.

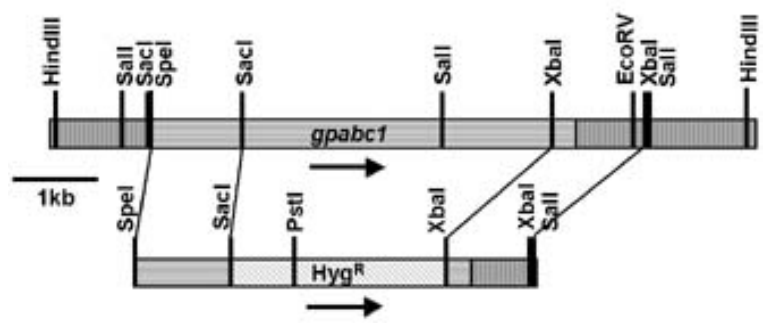

Fig. 2. Construction of the transformation vector pABCko. A 1,039-bp SacI fragment and a 1,035-bp XbaI fragment from the $5^{\prime}$ and $3^{\prime}$ regions, respectively, of the Gpabcl coding region were cloned into plasmid pGPC1 on either side of the hygromycin cassette. The linear SalI/SpeI fragment containing the hygromycin cassette flanked by these Gpabcl fragments was used for transformation. frame (ORF) that encoded a predicted protein of 1,491 amino acids. The ORF was interrupted by two introns of 47 and 56 $\mathrm{bp}$, and an additional 50-bp intron was located in the 5'-untranslated portion of the transcript. In a BLAST search, the gene, designated Gpabcl (accession number AJ306607), showed high homology to multidrug-resistance transporters of yeast and other fungi, especially to $A B C 1$ of Magnaporthe grisea (Urban et al. 1999). $A B C 1$ and Gpabcl share 65\% identity at the amino acid level and possess identical Walker motifs and ABC signature sequences (N-terminal: WalkerA GPPGSGCST, WalkerB QCWDNSTRGLD, ABC signature VSGGERKRVTIA. C-terminal: WalkerA GVSGAGTTL, WalkerB LFVDEPTSGLD, ABC signature NVEQRKRLTIGV).

Northern analysis of $G p a b c 1$ induction by rishitin revealed that the expression of the gene is induced within 5 to $10 \mathrm{~min}$ after addition of $150 \mu \mathrm{g}$ of rishitin per $\mathrm{ml}$ to mycelium in liquid culture (Fig. 1) and the transcript is detectable for at least $10 \mathrm{~h}$. A rishitin content of as little as $25 \mu \mathrm{g}$ per ml was still sufficient to turn on the transcription of the gene.

\section{Replacement of Gpabc1.}

In order to test the importance of the $\mathrm{ABC}$ transporter for tolerance to potato phytoalexins, we conducted gene replacement of Gpabcl. The replacement vector pABCko was constructed by cloning a 1,039-bp SacI fragment of the $5^{\prime}$ - region of $G p a b c 1$ and a 1,035-bp XbaI fragment of the 3'-region into the vector pGPC1 (Desjardins et al. 1992). The G. pulicaris wild-type isolate R6380 was transformed with a linear SalI/SpeI fragment containing the hygromycin cassette flanked by the $5^{\prime}$ - and $3^{\prime}$-transporter gene fragments (Fig. 2).

Transformants in which the internal part of the Gpabcl gene was replaced with the hygromycin cassette were identified by Southern analysis (Fig. 3). We obtained four different types of transformants. Of 55 transformants resulting from two independent transformation experiments, 24 had one or more ectopic integrations, as indicated by the presence of the $8-\mathrm{kb}$ wild-type fragments and one or more other hybridizing fragments (type 1, T47).

In 31 of the transformants, homologous integrations occurred at the Gpabcl locus. This was indicated by the replacement of the $8-\mathrm{kb}$ HindIII wild-type fragment by one or more fragments of different sizes. Two of these transformants contained the expected 7.1-kb fragment characteristic for a simple replacement of the internal part of the Gpabcl gene by the hygromycin cassette (type 2, T76) and an additional integration

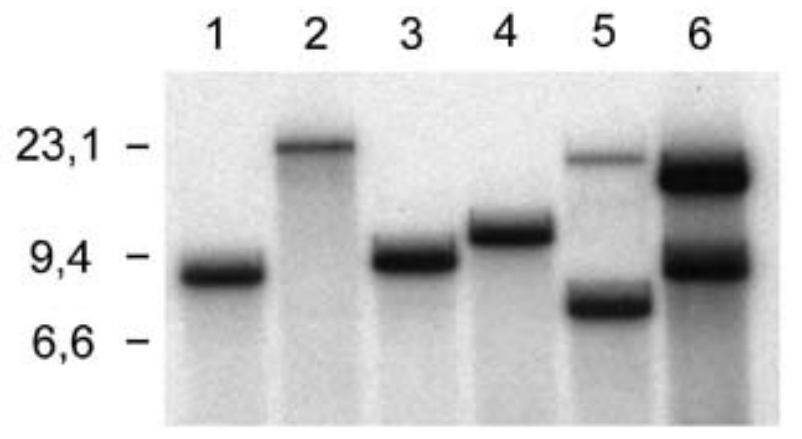

Fig. 3. Southern analysis of Gibberella pulicaris transformed with the Gpabc1-hygromycin cassette transformation fragment. HindIII-digested DNA of transformants was probed with the entire linear fragment used for transformation (Fig. 2). Lane 1, R6380 (wild type); lane 2, T30; lane 3, T74; lane 4, T77; lane 5, T76; and lane 6, T47. The wild type DNA contains the expected $8-\mathrm{kb}$ fragment. The hybridization pattern for T30, $\mathrm{T} 74$, and T77 indicates homologous integration of the transformation fragment with internal rearrangements. The pattern for T76 indicates both homologous and ectopic integration of the transformation fragment, while the pattern for T47 indicates ectopic integration only. 
event at an ectopic site. In all other transformants the single hybridizing HindIII fragment varied in size but was always larger than $7.1 \mathrm{~kb}$. The size of these HindIII fragments indicated either integration of several fragments at the Gpabcl locus, as in T30 (type 3), or rearrangement of the integrated DNA leading to a partial duplication within the integrated fragment (type 4, T74 and T77). Hybridizations of PstI-digested DNA of eight of these latter transformants with the complete transformation fragment detected three hybridizing bands in each lane (Fig. 4). Rehybridization of the Southern blot both with border fragments alone and with the hygromycin cassette revealed that the $6.6-\mathrm{kb}$ fragment represents the $5^{\prime}$ border and that the $3.2-\mathrm{kb}$ fragment represents the $3^{\prime}$ border (data not shown). These two fragments are present in the DNA of six transformants, whereas two transformants showed an increase in size of the $5^{\prime}$ PstI fragment. The DNA of all eight transformants contained an additional fragment that varied in size and specifically hybridized to the hygromycin probe. This variation in size indicated that the rearrangement occurred within the internal part of the transformation fragment involving the hygromycin cassette, possibly leading to a partial duplication of this gene. This result and the presence of the two PstI fragments of the correct size in six of the transformants prove that the sequences around the integration site are obviously unaffected, at least in these six transformants. Therefore, we chose two of these transformants (T74 and T77, type 4), one with several integrations at the homologous site (T30), and T76 with a perfect replacement and one additional ectopic integration for further experiments.

To determine whether gene replacement successfully inactivated $G p a b c 1$, northern analyses of the four representative replacement transformants (T76, type 2; T30, type 3; and T74 and T77, type 4) and one with an ectopic integration (T47, type

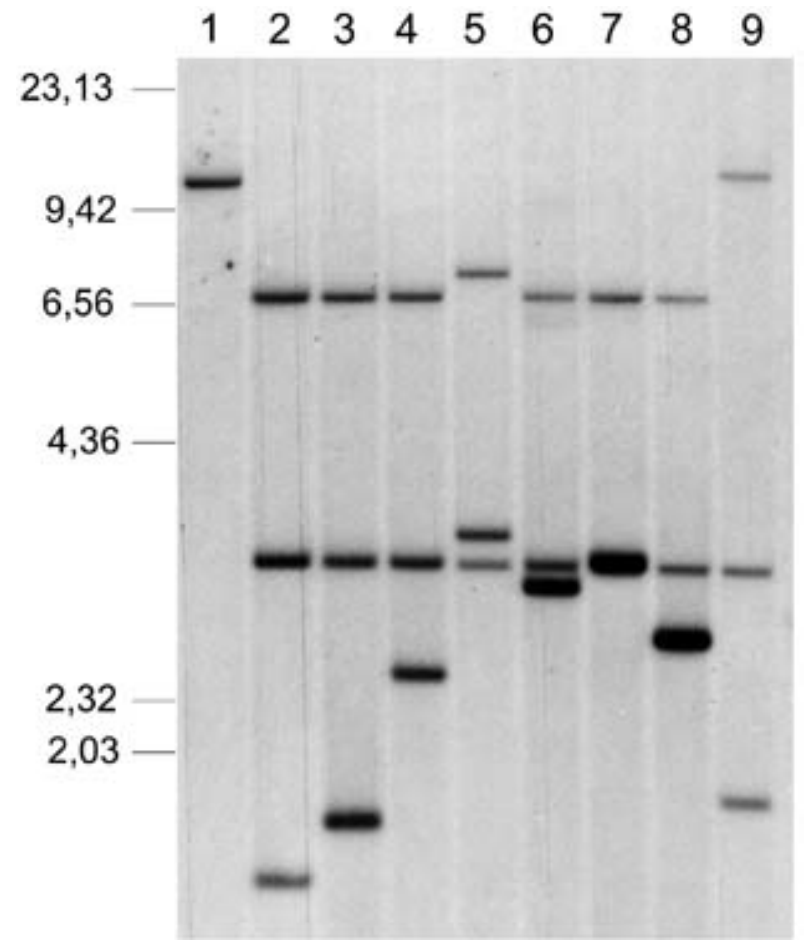

Fig. 4. Southern analysis of transformants in which the transformation fragment integrated only via homologous recombination at the Gpabcl locus (Fig. 3). To analyze the type of rearrangement within the integrated DNA, PstI-digested DNA of eight transformants was probed with the whole linear fragment used for transformation (Fig. 2). Lane 1, R6380 (wild type); lane 2, T74; lane 3, T75; lane 4, T77; lane 5, T91; lane 6, T101; lane 7, T108; lane 8, T115; and lane 9, T121.
1) were performed. After exposure to rishitin for $30 \mathrm{~min}$, the Gpabcl gene transcript was not detectable in the replacement mutants. The transformant with the ectopic integration showed gene expression identical to that of the wild type (Fig. 5). This analysis confirmed that the gene is not expressed at detectable levels in the transformants with a disrupted $G p a b c l$ gene.

\section{Phenotypic characterization of Gpabc1 replacement mutants.}

The four replacement mutants were analyzed for tolerance to rishitin by placing a mycelial plug on agar medium supplemented with $200 \mu \mathrm{g}$ of the phytoalexin per ml. As shown in Figure 6, growth of the mutants was dramatically reduced by $90 \%$ on rishitin-amended medium compared to the wild type after 3 days of incubation. On medium without rishitin, mutants and wild type grew equally well, covering the whole plate within the incubation time. Growth of the ectopic mutant T47 was the same as the wild type on both the amended and nonamended medium.

In two separate experiments, all transformants were tested for their ability to metabolize rishitin by inoculating mycelial mats of the transformants and R6380 on rishitin-amended Czapek-Dox agar medium for $24 \mathrm{~h}$. All transformants and the wild-type strain completely degraded the substrate within this time period, as indicated by the lack of rishitin in all agar extracts except for that of the noninoculated control plates (data not shown). This strongly suggests that the multidrug-resistance transporter encoded by Gpabcl is necessary for the tolerance of $G$. pulicaris to rishitin.

In order to test the impact of the inactivated $\mathrm{ABC}$ transporter gene on virulence of $G$. pulicaris on potato, all four replacement mutants, the ectopic transformant, and the wild-type progenitor strain R6380 were tested for their ability to colonize potato tuber tissue by placing a mycelial plug at the edge of a potato disc and incubating it for 5 days at $20^{\circ} \mathrm{C}$. As shown in Figure 7, the untransformed strain R6380 and the ectopic transformant colonized more than half of the potato slice. In contrast, growth of the Gpabcl replacement mutants was restricted to the inoculation site. Extended incubation did not result in further colonization of the potato slice by the mutants in contrast to strain R6380, which eventually macerated the entire tuber disc. Obviously, growth of the replacement mutants is completely stopped, which strongly suggests that the reduction in virulence is caused by the inability of the mutants to escape the toxic effect of rishitin by excreting the phytoalexin.

\section{Complementation of a Gpabc1 replacement mutant with the wild type gene.}

To obtain final proof that the observed phenotype was the result of the functional inactivation of the $\mathrm{ABC}$ transporter, we

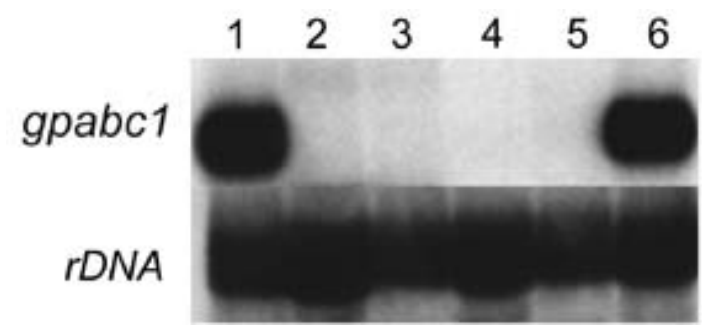

Fig. 5. Northern analysis of the Gpabcl replacement mutants. To test the expression of Gpabcl in mutant strains, total RNA was isolated from mycelia incubated for $30 \mathrm{~min}$ with rishitin $(150 \mu \mathrm{g} / \mathrm{ml})$ and probed with the 1,053-bp XbaI fragment of Gpabc1. Lane 1, R6380 (wild type); lane 2, T30; lane 3, T74; lane 4, T77; lane 5, T76; and lane 6, T47. The equal loading of each lane with $15 \mu \mathrm{g}$ of RNA per lane was verified by hybridization with a part of the rDNA cluster of Gibberella pulicaris. 
complemented one of the replacement transformants with a functional copy of Gpabcl. The complementation vector was constructed by cloning a genomic 8-kb HindIII fragment carrying the Gpabcl gene into pAN8-1 (Mattern et al. 1988), which contains a phleomycin resistance cassette. The mutant T74 was used as the recipient strain for the entire circular plasmid. A total of 43 transformants were tested for reconstitution of the wild-type phenotype by inoculation on potato tuber slices. After 5 days of incubation, several transformants had a virulence phenotype comparable to wild-type strain R6380 (Fig. 7). Three of these pathogenic transformants were tested for tolerance to rishitin. All three of them regained tolerance to the phytoalexin (Fig. 6). Northern analysis confirmed that the wild-type phenotypes were due to the expression of a functional Gpabcl gene. As shown in Figure 8, all three of the transformants expressed the gene in response to incubation with rishitin. These experiments clearly demonstrate that expression of a functional Gpabcl gene is necessary for both tolerance to rishitin and virulence on potato tubers.

\section{DISCUSSION}

Over the past few decades, much circumstantial evidence concerning the importance of phytoalexins for plant resistance against fungi has been produced. Genetic studies on the correlation between metabolism of phytoalexins and virulence have indicated that the tolerance of these compounds is essential for virulence of phytopathogenic fungi (Morrissey and Osbourn 1999; VanEtten et al. 1995). The best examples of this hypothesis involve the metabolism of pisatin and maackiain by $N$. haematococca and rishitin degradation by $G$. pulicaris (Desjardins et al. 1992; Miao and VanEtten 1992; VanEtten et al. 1989). In these fungi, high virulence is always correlated with the ability to metabolize phytoalexins. However, the isolation of $G$. pulicaris strains that were avirulent but able to metabolize rishitin indicated that there must be other genes, apart from metabolism genes, with a major impact on virulence (Desjardins and Gardner 1991). De Waard (1997) and Del Sorbo and associates (2000) proposed that such virulence

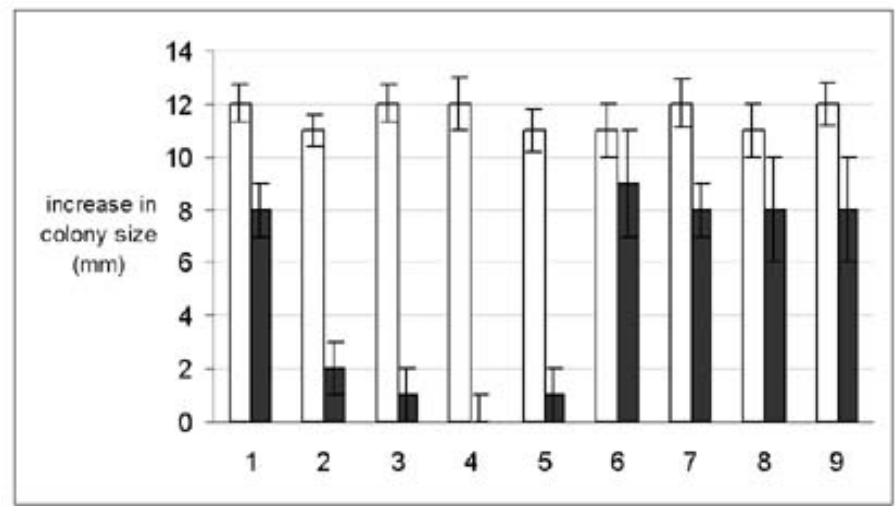

Fig. 6. Comparison of the tolerance to rishitin of wild type strain R6380 with Gpabcl replacement and complemented mutants. Mycelial plugs were placed at the center of Czapek-Dox agar plates without (control) and with rishitin $(200 \mu \mathrm{g} / \mathrm{ml})$ and incubated at $28^{\circ} \mathrm{C}$ in the dark. After 3 days, the increase in size of the colony was measured from the edge of the inoculating plug to the edge of the colony. Each value represents the mean \pm standard error of at least three replicates. Column 1, R6380 (wild type); column 2, T30; column 3, T76; column 4, T77; column 5, T74 (replacement mutants); column 6, T47 (ectopic); column 7, K4; column 8, K32; and column 9, K38 (complementation mutants). White bars = control. Black bars = on rishitin.
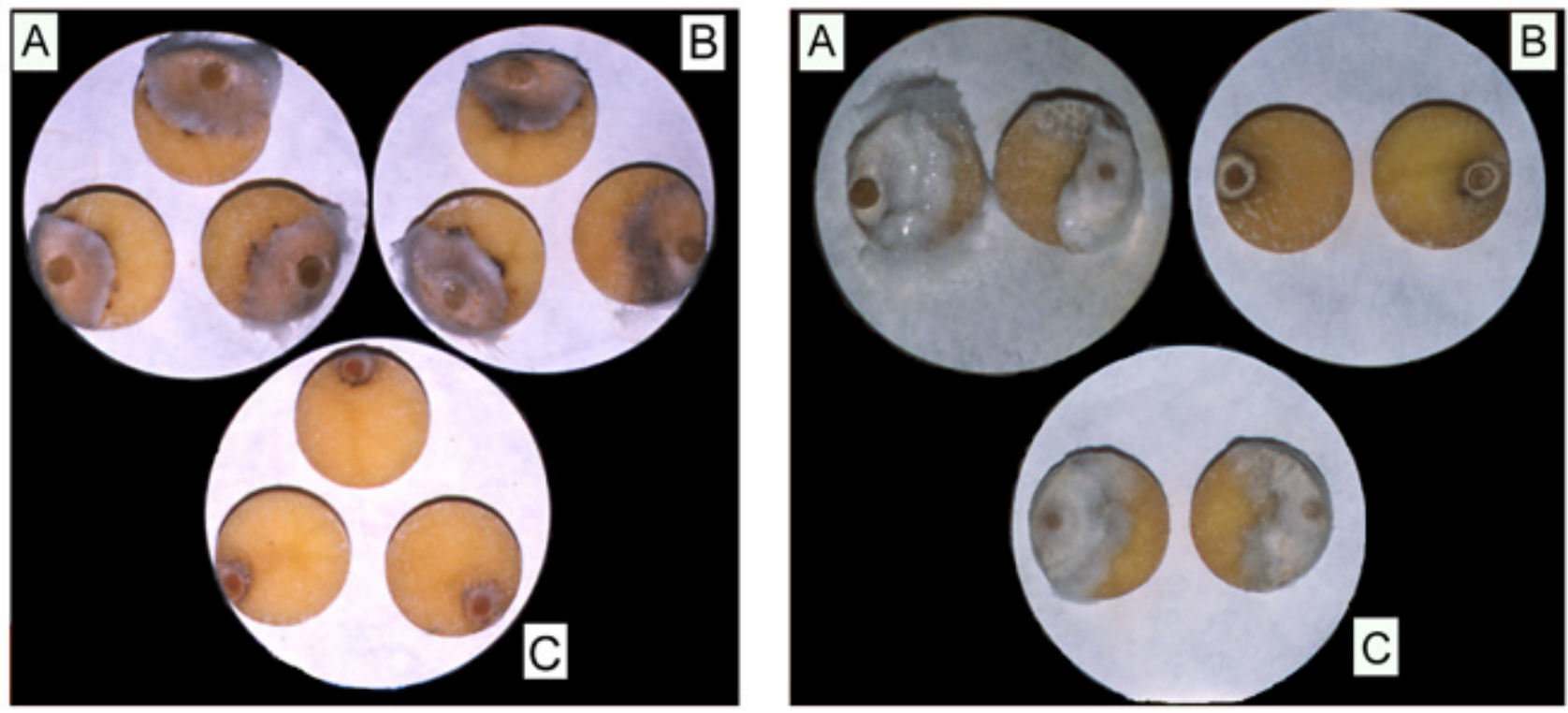

Fig. 7. Pathogenicity assay. Potato slices were inoculated with the following strains and incubated for 5 days at $20^{\circ} \mathrm{C}$. Left panel: A, R6380 (wild type); B, T47 (ectopic); C, T30 (replacement). Right panel: A, R6380 (wild type); B, T74 (replacement); C, K38 (complemented). 
genes may encode $\mathrm{ABC}$ multidrug-resistance transporters. They were able to support this theory to some extent by disrupting the $\mathrm{ABC}$ transporter gene BcatrB in $B$. cinerea. This gene is induced by the grapevine phytoalexin resveratrol, and its inactivation increased sensitivity to the phytoalexin and slightly reduced virulence of the fungus on grapevine leaves (Schoonbeek et al. 2001). The data on Gpabcl presented in this paper further verify this hypothesis and prove that multidrugresistance transporters not only contribute to, but can be essential for, the virulence of phytopathogenic fungi among the chemical defenses of their host plant. Therefore, these data are a very strong argument in favor of the phytoalexin theory, which proposes that phytoalexins are an important plant defense mechanism that has to be overcome by tolerance mechanisms of phytopathogenic fungi.

The Gpabcl gene is induced within minutes at the low threshold of $25 \mu \mathrm{g}$ of rishitin per $\mathrm{ml}$. This rapid and sensitive reaction of the fungus suggests that the function of the Gpabcl encoded transporter is the immediate export of phytoalexins from the infection hyphae. This enables the fungus to outgrow the area of wounded tissue in which these chemicals accumulate. In the replacement mutants, the lack of the transporter and the consequent toxic action of the phytoalexins causes arrest of mutant growth at the initial infection site. This reduced growth, in turn, may enable the host tissue to further restrict the growth of the fungus by activating additional defense reactions, such as lignification or accumulation of polyphenols.

The broad substrate range of $\mathrm{ABC}$ multidrug-resistance transporters renders it likely that the GPABC1 transporter also exports other potato phytoalexins, such as lubimin, solavetivone, or phytuberin. Preliminary experiments indicated that lubimin induces Gpabcl in the same manner as rishitin and that the Gpabcl replacement mutants became sensitive to this compound (A. Fleißner, unpublished data). Isolation of sufficient amounts of lubimin is under way to verify these results.

An ABC transporter is a perfect explanation for the nondegradative pisatin tolerance of $N$. haematococca described by Denny and VanEtten (1983a and 1983b; Denny et al. 1987), who found that tolerance of germinated spores of the fungus to the phytoalexin was mediated by an energy-dependent export mechanism and that the tolerance mechanism was inducible by other structurally related and unrelated substances. Both features are characteristic for $\mathrm{ABC}$ multidrug-resistance transporters, which need ATP as an energy source and usually have a very broad substrate range. In these studies, Denny and VanEtten already suggested that the nondegradative tolerance of phytoalexins may be more important than metabolism. This

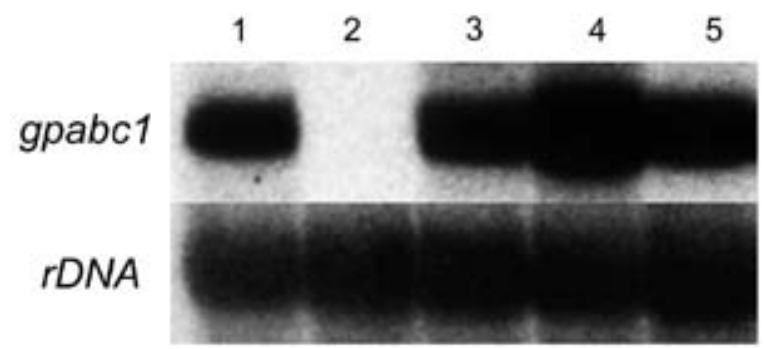

Fig. 8. Northern analysis of the complemented mutants. To test the expression of $G p a b c 1$ in mutant strains, total RNA was isolated from mycelium incubated for $30 \mathrm{~min}$ with rishitin $(150 \mu \mathrm{g} / \mathrm{ml})$ and probed with the replaced internal fragment of the Gpabc1 gene. Lane 1, R6380 (wild type); lane 2, T74; lane 3, K4; lane 4, K32; and lane 5, K38. The amount of RNA per lane was tested by hybridization with a part of the rDNA cluster of Gibberella pulicaris. early hypothesis is confirmed by our results, because the replacement transformants are inhibited in growth, although they are able to metabolize rishitin. This proves that export of the phytoalexin is a prerequisite for tolerance that cannot be substituted by metabolism. This conclusion is indirectly supported by experiments conducted by Enkerli and associates (1998) and Wasmann and VanEtten (1996) in which N. haematococca mutants deficient in PDAl and MAKl genes showed only a $30 \%$ reduction in growth compared with the wild type, indicating that, while the detoxification of phytoalexins contributes to tolerance, it is not the major factor.

Loss of virulence of a phytopathogenic fungus as a result of inactivation of an ABC transporter gene has already been described in $M$. grisea (Urban et al. 1999). In this fungus, replacement of the $A B C 1$ gene, which has the highest sequence homology to Gpabcl of G. pulicaris, caused a complete loss of virulence on rice plants. However, the $M$. grisea $A B C 1$ mutants did not show increased sensitivity to rice phytoalexins or several other chemicals that induced $A B C 1$ expression. The fact that inactivation of these two closely related $\mathrm{ABC}$ transporter genes leads to a marked loss of virulence indicates that $A B C$ transporters have potential as targets for specific fungicides. Analysis of the role of $\mathrm{ABC}$ transporters in the virulence of other fungi should shed light on how useful $\mathrm{ABC}$ transporters are as fungicide targets.

\section{MATERIALS AND METHODS}

\section{Strains, media, and growth conditions.}

G. pulicaris strain R6380 was obtained from A. Desjardins (Desjardins and Gardner 1989). For long-term storage and to produce spores, the fungus was grown on V8 juice agar (Stevens 1974). Liquid cultures were grown by inoculating spores from a 7- to 10-day-old V8 juice agar plate culture for 3 days at $28^{\circ} \mathrm{C}$ on a rotary shaker in $100 \mathrm{ml}$ of Mantle medium (Mantle and Nisbet 1976).

\section{Metabolism assays.}

For an assay of phytoalexin metabolism, a mycelial mat was transferred to Czapek-Dox agar supplemented with $150 \mu \mathrm{g}$ of rishitin per $\mathrm{ml}$ and incubated at $28^{\circ} \mathrm{C}$ for several hours as previously described (Weltring and Altenburger 1998). The mycelium was removed after $24 \mathrm{~h}$, and the agar medium was extracted with ethyl acetate. The organic phase was analyzed for residual substrate by gas-liquid chromatography as previously described (Gardner et al. 1988).

\section{Tolerance and virulence assays.}

Mycelial plugs from the growing margin of a radially grown culture were used as inoculum for both assays. Tolerance of rishitin was analyzed by placing mycelial plugs on Czapek-Dox agar supplemented with $200 \mu \mathrm{g}$ of rishitin per $\mathrm{ml}$ and determining the degree of inhibition of radial growth according to Desjardins and Gardner (1989). To measure virulence, the mycelial plug was placed on a potato tuber slice of the cv. Hansa and the assay was incubated at $20^{\circ} \mathrm{C}$ for 5 days.

\section{Fungal transformation.}

Transformation of G. pulicaris was carried out as previously described (Weltring 1995) using a linear SalI/SpeI fragment from the replacement vector pABCko. The fragment was isolated from preparative agarose gels by electroelution, using the Biotrap BT 1000 system (Schleicher und Schuell, Dassel, Germany) according to the supplier's instructions. In the complementation experiment, the entire circular complementation vector carrying the Gpabcl gene was used for transformation. 


\section{Gene libraries.}

The cosmid library was constructed by ligating $40-$ to $45-\mathrm{kb}$ genomic Sau3A fragments size-selected through a sucrose gradient into cosmid pGPC1 (Desjardins et al. 1992); standard procedures were applied (Sambrook et al. 1989). The library consisted of approximately $3.5 \times 10^{4} \mathrm{CFU}$. The cDNA library was prepared as described in Loser and Weltring (1998).

\section{DNA isolation and manipulation.}

Plasmid DNA was prepared using the JETstar Plasmid Midi Kit (Genomed, Bad Oeynnausen, Germany) according to the supplier's instructions. Sequences were determined using the Thermo Sequenase Cycle Sequencing Kit (Amersham Buchler, Braunschweig, Germany). Fungal DNA was isolated from mycelium harvested from liquid culture using the method described by Doyle and Doyle (1990). For Southern analysis, the DNA was digested with restriction enzymes (MBI Fermentas, St. Leon-Rot, Germany), blotted on nylon membrane Hybond $\mathrm{N}^{+}$(Amersham) in $0.4 \mathrm{M} \mathrm{NaOH}$, and hybridized with ${ }^{32} \mathrm{P}-\mathrm{la}-$ beled DNA probes under stringent conditions according to standard protocols (Sambrook et al. 1989).

\section{RNA isolation and manipulation.}

For northern analysis, RNA was prepared using the RNAgents Total RNA Isolation System (Promega, Manheim, Germany). After photometric quantification, $15 \mu \mathrm{g}$ of the isolated RNA per lane were separated on a denaturing gel, transferred to Hybond $\mathrm{N}^{+}$nylon membrane by downward blotting in $5 \times \mathrm{SSC}(1 \times \mathrm{SSC}$ is $0.15 \mathrm{M} \mathrm{NaCl}$ plus $0.015 \mathrm{M}$ sodium citrate) and $20 \mathrm{mM} \mathrm{NaOH}$ for $1.5 \mathrm{~h}$ and hybridized at $65^{\circ} \mathrm{C}$ as described by Church and Gilbert (1984).

To prepare radiolabeled induced $(+)$ and noninduced (-) cDNA, mRNA was isolated directly from lyophilized mycelium that had been incubated on Czapek-Dox agar with $(+)$ or without (-) rishitin with Dyna Beads (Dynal, Oslo, Norway) according to the supplier's instructions. Reverse transcription into cDNA was accomplished by standard protocols (Sambrook et al. 1989) in the presence of radiolabeled dCTP (Amersham).

\section{ACKNOWLEDGMENTS}

The authors thank S. Möller for excellent technical assistance, S. Moore for critically reading the manuscript, and the Deutsche Forschungsgemeinschaft for financial support.

\section{LITERATURE CITED}

Balzi, E., Wang, M., Leterme, S., Vandyck, L., and Goffeau, A. 1994. PDR5, a novel yeast multidrug-resistance-conferring transporter controlled by the transcription regulator PDR1. J. Biol. Chem. 269:22062214.

Church, G. M., and Gilbert, W. 1984. Genomic sequencing. Proc. Natl. Acad. Sci. U.S.A. 81:1991-1995.

Covert, S. F., Enkerli, J., Miao, V. W., and VanEtten, H. D. 1996. A gene for maackiain detoxification from a dispensable chromosome of $\mathrm{Nec}$ tria haematococca. Mol. Gen. Genet. 251:397-406.

Cruickshank, I. A. M. 1962. Studies on phytoalexins IV: The antimicrobial spectrum of pisatin. Aust. J. Biol. Sci. 15:147-159.

Cruickshank, I. A. M., and Perrin, D. R. 1965. Studies on phytoalexins VIII: The effect of some factors on the formation, stability, and localization of pisatin in vivo. Aust. J. Biol. Sci. 18:817-828.

De Waard, M. A. 1997. Significance of ABC transporters in fungicide sensitivity and resistance. Pestic. Sci. 51:271-275.

Del Sorbo, G., Andrade, A. C., Van Nistelrooy, J. G., Van Kan, J. A., Balzi, E., and De Waard, M. A. 1997. Multidrug resistance in Aspergillus nidulans involves novel ATP-binding cassette transporters. Mol. Gen. Genet. 254:417-426.

Del Sorbo, G., Schoonbeek, H.-J., and De Waard, M. A. 2000. Fungal transporters involved in efflux of natural toxic compounds and fungicides. Fungal Genet. Biol. 30(1):1-15.
Denny, T. P., and VanEtten, H. D. 1983a. Characterization of an inducible, nondegradative tolerance of Nectria haematococca MP VI to phytoalexins. J. Gen. Microbiol. 129:2903-2913.

Denny, T. P., and VanEtten, H. D. 1983b. Tolerance of Nectria haematococca MP VI to the phytoalexin pisatin in the absence of detoxification. J. Gen. Microbiol. 129:2903-2913.

Denny, T. P., Matthews, P. S., and VanEtten, H. D. 1987. A possible mechanism of nondegradative tolerance of pisatin in Nectria haematococca MP VI. Physiol. Plant Path. 30:93-107.

Desjardins, A. E., and Gardner, H. W. 1989. Genetic analysis in Gibberella pulicaris: Rishitin tolerance, rishitin metabolism, and virulence on potato tubers. Mol. Plant-Microbe Interac. 2:26-34.

Desjardins, A. E., and Gardner, H. W. 1991. Virulence of Gibberella pulicaris on potato tubers and its relationship to a gene for rishitin metabolism. Phytopathology 81:429-435.

Desjardins, A. E., Gardner, H. W., and Weltring, K. M. 1992. Detoxification of sesquiterpene phytoalexins by Gibberella pulicaris (Fusarium sambucinum) and its importance for virulence on potato tubers. J. Ind. Microbiol. 9:201-211.

Doyle, J. J., and Doyle J. L. 1990. Isolation of plant DNA from fresh tissue. Focus 12:13-15.

Ebel, J. 1986. Phytoalexin synthesis: The biochemical analysis of the induction process. Annu. Rev. Phytopathol. 24:235-264.

Endicott, J. A., and Ling, V. 1989. The biochemistry of P-glycoproteinmediated multidrug resistance. Annu. Rev. Biochem. 58:137-171.

Enkerli, J., Bhatt, G., and Covert, S. F. 1998. Maackiain detoxification contributes to the virulence of Nectria haematococca MP VI on chickpea. Mol. Plant-Microbe Interac. 11:317-326.

Gardner, H. W., Desjardins, A. E., Weisleder, D., and Plattner, R. D. 1988. Biotransformation of the potato phytoalexin lubimin by Gibberella pulicaris: Identification of major products. Biochem. Biophys. Acta 966:347-356.

Gottesman, M. M., and Pastan, I. 1993. Biochemistry of multidrug resistance mediated by the multidrug transporter. Annu. Rev. Biochem. 62:385-427.

Hyde, S. C., Emsley, P., Hartshorn, M. J., Mimmack, M. M., Gileadi, U., Pearce, S. R., Gallagher, M. P., Gill, D. R., Hubbard, R. E., and Higgins, C. F. 1990. Structural model of ATP-binding proteins associated with cystic fibrosis, multidrug resistance, and bacterial transport. Nature 346:362-366.

Kuc, J. 1982. Phytoalexins from the Solanaceae. Pages 81-105 in: Phytoalexins. J. A. Bailey and J. W. Mansfield, eds. John Wiley and Sons, New York.

Loser, K., and Weltring, K. M. 1998. Induction of a polyubiquitin gene (ubil) by potato phytoalexins and heat shock in Gibberella pulicaris. Curr. Gen. 34:404-409

Mantle, P. G., and Nisbet L. J. 1976. Differentiation of Claviceps purpurea in axenic culture. J. Gen. Microbiol. 93:321-334

Mattern, I. E., Punt, P. J., and van den Hondel, C. A. M. J. J. 1988. A vector for Aspergillus transformation conferring phleomycin resistance. Fungal Genet. Newsl. 35:25

Miao, V. P. W., and VanEtten, H. D. 1992. Three genes for metabolism of the phytoalexin maackiain in the plant pathogen Nectria haematococca: Meiotic instability and relationship to a new gene for pisatin demethylase. Appl. Environ. Microbiol. 58:801-808.

Morrissey, J. P., and Osbourn A. E. 1999. Fungal resistance to plant antibiotics as a mechanism of pathogenesis. Microbiol. Mol. Biol. Rev. 63:708-724.

Müller, K. O., and Börger, H. 1940. Experimentelle Untersuchungen über die Phytophthora-Resistenz der Kartoffel. Arb. Biol. Anst. Reichsanst: (Berlin) 23:189-231.

Sambrook, J., Fritsch E. F., and Maniatis T. 1989. Molecular Cloning: A Laboratory Manual. 2nd ed. Cold Spring Harbor Laboratory Press, Cold Spring Harbor, NY, U.S.A.

Schoonbeek, H., Del Sorbo, G., and De Waard, M. A. 2001. The ABC transporter BcatrB affects the sensitivity of Botrytis cinerea to the phytoalexin resveratrol and the fungicide fenpiclonil. Mol. Plant-Microbe Interac. 14:562-571.

Seelig, A. 1998. A general pattern for substrate recognition by P-glycoprotein. Eur. J. Biochem. 251:252-261.

Stevens, R. B. 1974. Mycology Guidebook. Page 703. University of Washington Press, Seattle, WA. U.S.A.

Urban, M., Bhargava, T., and Hamer, J. E. 1999. An ATP-driven efflux pump is a novel pathogenicity factor in rice blast disease. EMBO (Eur. Mol. Biol. Organ.) J. 18(3):512-521.

VanEtten, H. D., Matthews, D. E., and Matthews, P. S. 1989. Phytoalexin detoxification: Importance for pathogenicity and practical implications. Annu. Rev. Phytopathol. 27:143-164.

VanEtten, H. D., Sandrock, R. W., Wasmann, C. C., Soby, S. D., McCluskey, K., and Wang, P. 1995. Detoxification of phytoanticipins 
and phytoalexins by pathogenic fungi. Can. J. Bot. 73(Suppl.1):518525.

Wasmann, C. C., and VanEtten, H. D. 1996. Transformation-mediated chromosome loss and replacement of a gene for pisatin demethylase decrease the virulence of Nectria haematococca on pea. Mol. PlantMicrobe Interac. 9:793-803.

Walker, J. E., Saraste, M., Runswick, M. J., and Gay, N. J. 1982. Distantly related sequences in the $\alpha$ - and $\beta$-subunits of ATP synthase, myosin, kinases, and other ATP-requiring enzymes and a common nucleotide binding fold. EMBO (Eur. Mol. Biol. Organ.) J. 1:945-951.
Weltring, K. M. 1995. A method for easy isolation of promotor fragments from promotor-probe libraries of filamentous fungi. Curr. Gen. 28:190196.

Weltring, K. M., and Altenburger, M. 1998. Metabolism of the phytoalexin rishitin in Gibberella pulicaris is highly reduced in liquid culture. Z. Naturforsch Sect. C Biosci. 53:806-810.

Weltring, K. M., Turgeon, B. G., Yoder, O. C., and VanEtten, H. D. 1988. Isolation of a phytoalexin-detoxification gene from the plant pathogenic fungus Nectria haematococca by detecting its expression in $\mathrm{As}$ pergillus nidulans. Gene 68:335-344. 